\title{
Acknowledgments
}

The following colleagues have generously given their time and energy to review papers for Law and Human Behavior over the past 12 months. We are grateful for their contributions to this journal.

\author{
Karen Abram \\ Fabiana Alceste \\ Apryl Alexander \\ Jaime Anderson \\ John Richard Anderson \\ Paul Appelbaum \\ Keisha April \\ Shelby Arnold \\ Karl Ask \\ Jennifer L. Beaudry \\ Gershon Ben-Shakkar \\ Vanessa Bohns \\ Ben Bradford \\ Neil Brewer \\ Laure Brimbal \\ Michael R. Brubacher \\ Kaila C. Bruer \\ Shawn Bushway \\ Jason Cantone \\ Curt Carlson \\ Caitlin Cavanagh \\ Edward C. Chang \\ Ted Chiricos \\ Annette Christy \\ Steven E. Clark \\ Hayley M. D. Cleary \\ Samantha Clinkinbeard \\ Adam Coffey \\ Simon Cole \\ Jennifer Cox \\ Angela M. Crossman \\ Melissa de Vel-Palumbo \\ Grace Deason \\ Matt DeLisi \\ Matthew DeMichele \\ Dennis J. Devine \\ Jason J. Dickinson \\ Diana Dolliver \\ Itiel Dror \\ Misty Christina Duke \\ James Dunlea \\ Jennifer Dysart \\ Joseph Eastwood \\ John Edens \\ Vanessa A. Edkins \\ Mitchell Eisen \\ Jacqueline Evans \\ Andrew Joseph Evelo
}

Jaymes Fairfax-Columbo

Amanda M. Fanniff

Neal Feigenson

Elise Fenn

Klaus Fiedler

Adam D. Fine

Ron Fisher

Heather Flowe

Dana Formon

Elizabeth Foster

Erika Fountain

Elizabeth Gale-Bentz

Michele Galietta

Meghann Patricia Galloway

Linda Geven

Deborah Goldfarb

Lauren Gonzales

W. Neil Gowensmith

Yael Granot

Rachel Leigh Greenspan

Laura Grossi

Lucy Guarnera

Angelica Hagsand

Joseph Hamm

Emily Haney-Caron

Valerie P. Hans

Maria Hartwig

Samuel Hawes

Rebecca K. Helm

Kelsey Henderson

Paul Hirschfield

Kathryn Holland

Lorraine Hope

Amelia Hritz

Jonathan Jackson

Christopher Jaeger

Sandy Jung

Kimberly Kaiser

Shannon Kelley

Sharon Kelley

Christopher E. Kelly

Patrick J. Kennealy

Steve Kleinman

Lauren E. Kois

Casey LaDuke

James Michael Lampinen

Lonn Lanza-Kaduce
G. Daniel Lassiter

Len Bruno Lecci

Richard Leo

Elisabeth Leroux

Benjamin R. Locklair

Timothy Luke

Mona Lynch

Thomas D. Lyon

Stephanie Madon

Jamal K. Mansour

Jaume Masip

Jason Matejkowski

James McCafferty

Kimberley A. McClure

Kelly McWilliams

Lauren Meaux

Ewout Meijer

Alyssa Mikytuck

Ojmarrh Mitchell

Kathryn Lynn Modecki

Andreas Mokros

Merry Morash

Richard K. Moule

Elyse Mowle

Robert Nash

Amanda NeMoyer

Anna-Kaisa Newheiser

Christopher J. Normile

Jill Norvilitis

Narina Nunez

Verena Andrea Oberlader

Mark E. Olver

Kevin O'Neil

Matthew Palmer

Marc Patry

Jennifer H. Peck

Emily Pica

Kerri L. Pickel

Justin Tyler Pickett

Alex Piquero

Maciej Michal Próchnicki

Jeffrey Rachlinski

Dana Radatz

Krystia Reed

Tara N. Richards

Christina Riggs Romaine

Richard Rogers
Jared Ruchensky

Mark Ruiz

Brenda Russell

Christine L. Ruva

Jason Rydberg

Anna Sagana

Randall T. Salekin

Travis Seale-Carlisle

Stephane M. Shepherd

Amy E. Smith

Andrew Michael Smith

Brian C. Smith

Roseanna Sommers

Barbara A. Spellman

Marissa Stanziani

Nancy Kay Steblay

Tim Stickle

Matthew Stimmel

Heidi Strohmaier

Jessica Sutherland

Melanie Takarangi

Paul Taylor

William C. Thompson

Alice Thornewill

Laura Catherine Thornton

Colin Getty Tredoux

Rick Trinkner

Siny Tsang

Jonathan P. Vallano

Bruno Verschuere

Stacy A. Wetmore

Lawrence T. White

Richard L. Wiener

Miko M. Wilford

Twila Wingrove

Roselle Wissler

Philip Witt

Skye A. Woestehoff

Scott E. Wolfe

Mary Wood

William Douglas Woody

Rachel Zajac

Charles Zapata

David Zimmerman

Mircea Zloteanu

Tina M. Zottoli 
The Editor and Associate Editors would like to express their sincere appreciation to members of the 2020 Student Editorial Board and Reviewer Mentee Program who have generously given their time and energy to review manuscripts for Law and Human Behavior.

\section{Student Editorial Board}

Rebekah Adair

Brittney Amber Maria Aparcero-Suero

Nydia Teresa Ayala

Kimberly M. Bernstein

Anna Grace Burnette

Jean Cabell

Mary Catlin

Ryan E. Ditchfield

Elizabeth Elliott

Tallie Armstrong Hoskowitz

Cassandra Bailey

Antonella Bariani

Joshua Behl

Brenna L. Giordano

Bethany Growns
Melanie B. Fessinger

Joshua Francis

Megan Giroux

Lauren Grove

Madison B. Harvey

Katherine Hazen

Lindsay Healey

Maria Jimenez

Ashley Jones

Allison Kurpiel
Samantha Luna

Mikaela Magnusson

Juliana Malico

Kureva Matuku

Kristen McCowan

Sarah A. Moody

Kendra Paquette

Christina Perez

Rachael T. Perrault

Hannah Phalen

Andrea Riederer
Lillian Rodriguez-Steen Jesse Rothweiler

Ryan A. Schneider

R. Taylor Stevens

Therese Todd

Kristina Todorovic

Gabriele Trupp

Jennifer Weintraub

\section{Reviewer Mentee Program}

Cassidy Haigh

Jennifer L. Harrison

Johanna Hellgren

Tamara Kang

Monica Lawson

Alexandra Lugo

\author{
Stefanie McLaney \\ Quincy Claire Miller \\ Sara Byrd Millspaugh \\ Emily M. Carstens Namie \\ Chelsey Parker \\ Joshua J. Reynolds \\ Andrea Wolfs
}

Alexandra Zidenberg 